\title{
Polarimetry of evolved stars
}

\section{The carbon star R Scl}

\author{
R. V. Yudin ${ }^{1,2}$ and A. Evans ${ }^{3}$ \\ 1 Central Astronomical Observatory of the Russian Academy of Sciences at Pulkovo, 196140 Saint-Petersburg, Russia \\ e-mail: ruslan61@hotmail.com \\ 2 Isaac Newton Institute of Chile, St.-Petersburg Branch \\ 3 Department of Physics, Keele University, Keele, Staffordshire, ST5 5BG, UK
}

Received 24 January 2002 / Accepted 3 June 2002

\begin{abstract}
We present broadband optical polarimetry of the carbon star R Scl, primarily in the $V R_{\mathrm{C}} I_{\mathrm{C}}$ bands. Polarimetric variability was detected on time-scales from hours to years. The polarization of R Scl showed a $\sim \lambda^{-4}$ wavelength-dependence, which we attribute to scattering by small amorphous carbon dust grains. We deduce the properties of the circumstellar condensations in which the scattering takes place, and find consistency with infrared data if the condensations move in eccentric orbits. We also conclude that the condensations are tidally dispersed on a time-scale $\sim 1$ day. On longer time-scales, polarimetric variations may possibly be linked with photometric variations.
\end{abstract}

Key words. polarization - stars: circumstellar matter - stars: carbon - stars: individual: R Scl - stars: variables: general

\section{Introduction}

\subsection{General remarks}

One of the unsolved problems of the astrophysics of evolved stars is the mechanism whereby ostensibly spherical stars, when they shed their envelopes during post-AGB evolution, make the transition to spectacularly aspherical planetary nebulae. While direct imaging of scattered radiation from the circumstellar (CS) environments of evolved stars is now being carried out (e.g. Monnier et al. 2000; Gledhill et al. 2001), to date the number of objects observed in this way is limited.

However, in the absence of direct imaging, polarization of the star (and its time- and wavelength-dependence) prior to shedding its envelope is a powerful and under-utilized tool for probing the distribution of matter, and hence mass ejection mechanisms (e.g. Johnson \& Jones 1991). For example, if the star is a component of a binary system, we might expect consequent asymmetries or inhomogeneities in the CS envelope, or anisotropic illumination; in either case the radiation is polarized. Even if binarity is not a factor, nascent asymmetries can be probed polarimetrically, leading to information about the distribution of dust, and geometry of mass-loss.

The case of the carbon star R Scl is particularly significant, as polarimetric measurements can be correlated with direct observations of the scattered light from the CS envelope (González Delgado et al. 2001), and the interpretation of polarimetric observations is not carried out in isolation.

Send offprint requests to: A. Evans, e-mail: ae@astro.keele.ac.uk
R Scl effectively provides a "test case" for the interpretation of polarimetric observations of mass-loss from evolved stars.

\section{2. $R \mathrm{Scl}$}

R Scl (= HD 8879, HR 423, IRAS 01246-3248, HIP 6759, spectral type $\mathrm{C} 6,5)$ is a well-studied carbon-rich $(\mathrm{C} / \mathrm{O} \simeq 1.4)$ AGB star; its effective temperature varies between $2930 \mathrm{~K}$ at light curve minimum to $2650 \mathrm{~K}$ at maximum (Hron et al. 1998). It is a pulsational variable, and the photometric period of $373^{\mathrm{d}} .6$ is generally considered to be a period of pulsation (Whitelock et al. 1997). It seems that the majority of oxygenrich Miras pulsate in the first overtone, while one carbon-rich Mira (R Lep) pulsates in the fundamental (see Whitelock et al. 1997 and references therein). In the case of R Scl the situation is not clear: the contibution of the "first harmonic" to the light variations is complicated by the fact that the "first harmonic" is very close to the one-year alias of the "fundamental". There is another periodicity, $\simeq 1804$ days, in the variability of $\mathrm{R} \mathrm{Scl}$, the origin of which is unknown (Whitelock et al. 1997).

The somewhat unusual IRAS colours of R Scl indicate the presence of two dust shells, a warm shell close to the star and a cool, more distant, shell. The ISO spectrum (Hron et al. 1998) is rich in hydrocarbon molecules, and emission by silicon carbide dust.

$\mathrm{R}$ Scl has an extended shell, detected by sub-millimetre and radio observations (see, for example, Olofsson et al. 2000). González Delgado et al. (2001) have detected extended 
emission around $\mathrm{R} \mathrm{Scl}$ in the $\mathrm{K} \mathrm{I}$ and $\mathrm{NaD}$ lines; they conclude that the scattered light is due to Rayleigh scattering by small grains, possibly located in the CO-bearing shells detected in the sub-millimetre and radio (Olofsson et al. 1996). Whitelock et al. (1997) have estimated the mass-loss from R Scl using a wind velocity of $20 \mathrm{~km} \mathrm{~s}^{-1}$ and the IRAS $60 \mu \mathrm{m}$ flux; they find $\dot{M} \simeq 1.55 \times 10^{-6} M_{\odot} \mathrm{yr}^{-1}$, an order of magnitude higher than that implied $\left(\sim 1.6 \times 10^{-7} M_{\odot} \mathrm{yr}^{-1}\right)$ by the empirical relationship between $\dot{M}$ and pulsation period for Galactic Miras (see Vassiliadis \& Wood 1993).

The distance of $\mathrm{R} \mathrm{Scl}$ as determined by HIPPARCos is $474 \mathrm{pc}$ (Alksnis et al. 1998); assuming visual interstellar (IS) extinction of $0.1 \mathrm{mag}$, the absolute visual and bolometric magnitudes are -1.91 and -6.09 respectively.

The pulsation period and absolute bolometric magnitude enable us to make a crude estimate of the mass of $\mathrm{R} \mathrm{Scl}$, or at least of that of its progenitor. Vassiliadis \& Wood (1993) have carried out a study of the post-main sequence evolution of stars with initial masses in the range $0.9<M / M_{\odot}<5$, with a range of metallicities. Their Fig. 20, displaying the theoretical relationship between absolute bolometric magnitude and pulsational period for the quiescent $\mathrm{H}$-burning phase of a shell flash, suggests a mass of $\sim 4 M_{\odot}$ for the progenitor of R Scl. We have no direct information on how much mass $\mathrm{R} \mathrm{Scl}$ has lost since it was on the main sequence; however taking the lifetimes for the various post-main sequence phases for a $\sim 4 M_{\odot}$ star in Vassiliadis \& Wood (1993) and the above mass-loss rate, the mass lost is unlikely to exceed $1 M_{\odot}$, and is most likely much less than this. Accordingly for our present purposes we assume that the mass of $\mathrm{R} \mathrm{Scl}$ is $M=3.5 M_{\odot}$.

There has been little study of the variations in polarization with light curve phase for stars of this kind, and in particular of the long-term polarimetric variations. Magalhães et al. (1986) have carried out a four-year study of the semi-regular variable $L_{2}$ Pup and found evidence for changes in both magnitude and position angle (PA) of polarization, and its wavelengthdependence. Raveendran \& Rao (1989) have studied the carbon Mira R Lep; they also find significant changes in the degree, PA and wavelength-dependence of polarization.

In this paper we present new polarization measurements of $\mathrm{R} \mathrm{Scl}$. We combine these with data from Serkowski \& Shawl (2001) to (i) investigate the temporal variability, on different time-scales, on the $q u$ diagram, (ii) study the wavelengthdependence of polarization, (iii) look for possible correlations of polarization with light curve phase and (iv) consider briefly possible mechanisms which might be responsible for the observed behaviour.

\section{Observations and analysis}

The observations of $\mathrm{R} \mathrm{Scl}$ presented here were taken through $V R_{\mathrm{C}} I_{\mathrm{C}}$ filters and, on one occasion, the $B$-filter using the UCT polarimeter (Cropper 1985); all observations were made with a $20^{\prime \prime}$ aperture. The data were obtained in two seasons in 1994 and 1995 during five nights, in principle allowing us to study its temporal variability on short and long time-scales. Full details of the observations and analysis have been presented elsewhere (Clarke et al. 1998; Clarke \& Stewart 1986;
Yudin \& Evans 1998, 2002) and are not repeated here. The data are listed in Table 1, in which the Julian days are the time of mid-observation.

The values of the normalized Stokes parameters (NSP) $q=Q / I$ and $u=U / I$ in Table 1 were corrected for instrumental polarization, noise bias etc. using the formalism of Clarke \& Stewart (1986). Where polarization is detected at the $99 \%(\sim 3 \sigma)$ confidence level the values of the degree of polarization $P$ and equatorial PA $\theta$ are given in Table 1 . The NSP (corrected for instrumental polarization) were also used to test for variability. The detection level of circular polarization $\left(P_{\text {circ }} \equiv v=V / I\right)$ is shown in the last column in Table 1. Estimates of the visual magnitudes (with accuracy $\pm 0^{\mathrm{m}} .1$ ) of the program star, obtained with the UCT polarimeter, are also given in Table 1.

For the purposes of determining light curve phase, we use $2450319.02+373.6 \times E$,

which we have calculated from the data of Hron et al. (1998). On the longer time-scale, our data were separated by about $344^{\mathrm{d}}-358^{\mathrm{d}}$, close to the pulsation period. The object was therefore observed at approximately the same photometric phase (0.00-0.01 and 0.95-0.96 for the 1994 and 1995 seasons respectively), close to maximum light (cf. the infrared light curve by Whitelock et al. 1997; other than the sparse visual light curve in the HIPPARCos data base we are not aware of an equivalent visual light curve). Therefore while our SAAO data by themselves do not allow us to look for correlations between polarimertic variability and phase, they do enable us to consider variations on 1-year and shorter time-scales.

\section{Discussion}

Our data indicate that $\mathrm{R} \mathrm{Scl}$ shows detectable polarization at the $\simeq 0.5 \%$ level. We estimate the IS component of polarization in the $V$-band using the data in Heiles (2000), together with the distance to the object as derived from the HIPPARcos parallax, to be $P_{\text {is }} \approx 0.2 \%$ at $\theta_{\text {is }} \approx 157^{\circ}$. After correction for the IS component, we find that intrinsic polarization is present at the $99.9 \%$ confidence level. The mean value of intrinsic polarization is about $0.2-0.3 \%$ in the $V R_{\mathrm{C}} I_{\mathrm{C}}$ bands.

In the following we discuss the polarimetric variability, and the wavelength-dependence of polarization.

\subsection{Polarimetric variability}

\subsubsection{Variability within a pulsation cycle}

The Stokes parameters are plotted in the $q u$-plane in Fig. 1a. It is evident that $\mathrm{R} \mathrm{Scl}$ displays polarimetric variability in all the investigated passbands on different time-scales, from hours through days to a year. This variability was detected at the 99.9\% confidence level.

Earlier polarimetric measurements of $\mathrm{R} \mathrm{Scl}$ obtained in 1966-1969 were published recently by Serkowski \& Shawl (2001). Our data, combined with those of Serkowski \& Shawl (2001) (see Fig. 1b), allow us to study the behaviour in the $q u$ plane in more detail. Inspection of Fig. 1b suggests 
Table 1. Polarimetric data for R Scl.

\begin{tabular}{rrrrcccrrr}
\hline \hline Object & JD & Filter & $m_{V}$ & $q(\%)$ & $u(\%)$ & $P(\%)$ & $\theta\left(^{\circ}\right)$ & $v(\%)$ & $\begin{array}{c}P_{\mathrm{c}} \\
\text { level }\end{array}$ \\
\hline R Scl & $579000+$ & & & & & & & \\
& 571.610 & $V$ & 5.6 & & & & & & \\
& 571.612 & $R_{\mathrm{C}}$ & & $0.164 \pm 0.034$ & $-0.303 \pm 0.034$ & $0.345 \pm 0.034$ & $149.2 \pm 2.8$ & $0.036 \pm 0.024$ \\
& 571.615 & $I_{\mathrm{C}}$ & & $0.316 \pm 0.027$ & $-0.167 \pm 0.027$ & $0.357 \pm 0.027$ & $166.1 \pm 2.2$ & $-0.013 \pm 0.019$ \\
& 571.626 & $V$ & 5.6 & $0.255 \pm 0.106$ & $-0.575 \pm 0.106$ & $0.629 \pm 0.106$ & $147.0 \pm 4.8$ & $-0.006 \pm 0.075$ \\
& 571.627 & $R_{\mathrm{C}}$ & & $-0.063 \pm 0.098$ & $-0.499 \pm 0.098$ & $0.503 \pm 0.098$ & $131.4 \pm 5.6$ & $0.093 \pm 0.069$ \\
& 571.628 & $I_{\mathrm{C}}$ & & $0.305 \pm 0.079$ & $-0.091 \pm 0.079$ & $0.319 \pm 0.079$ & $171.7 \pm 7.1$ & $-0.079 \pm 0.056$ \\
& 571.634 & $V$ & 5.6 & $0.218 \pm 0.061$ & $-0.150 \pm 0.061$ & $0.265 \pm 0.061$ & $162.7 \pm 6.6$ & $-0.065 \pm 0.043$ \\
& 571.637 & $R_{\mathrm{C}}$ & & $0.240 \pm 0.035$ & $-0.369 \pm 0.035$ & $0.440 \pm 0.035$ & $151.5 \pm 2.3$ & $-0.072 \pm 0.025$ & $2.9 \sigma$ \\
& 575.643 & $B$ & & $0.712 \pm 0.380$ & $-0.454 \pm 0.380$ & $0.844 \pm 0.380$ & $163.7 \pm 13$ & $-0.041 \pm 0.269$ \\
& 575.645 & $V$ & 5.7 & $0.271 \pm 0.059$ & $-0.151 \pm 0.059$ & $0.310 \pm 0.059$ & $165.4 \pm 5.5$ & $0.088 \pm 0.042$ & $2.1 \sigma$ \\
& 575.648 & $R_{\mathrm{C}}$ & & $0.314 \pm 0.033$ & $-0.174 \pm 0.033$ & $0.359 \pm 0.033$ & $165.5 \pm 2.6$ & $-0.015 \pm 0.023$ \\
& 575.653 & $I_{\mathrm{C}}$ & & $0.431 \pm 0.047$ & $-0.107 \pm 0.047$ & $0.444 \pm 0.047$ & $174.0 \pm 3.0$ & $-0.016 \pm 0.033$ \\
& 579.639 & $V$ & 5.7 & $0.453 \pm 0.073$ & $-0.232 \pm 0.073$ & $0.509 \pm 0.073$ & $166.4 \pm 4.1$ & $0.101 \pm 0.051$ & $2.0 \sigma$ \\
& 579.641 & $R_{\mathrm{C}}$ & & $0.276 \pm 0.040$ & $-0.113 \pm 0.040$ & $0.298 \pm 0.040$ & $168.9 \pm 3.8$ & $0.049 \pm 0.028$ & $1.8 \sigma$ \\
& 579.642 & $I_{\mathrm{C}}$ & & $0.362 \pm 0.028$ & $-0.156 \pm 0.028$ & $0.394 \pm 0.028$ & $168.3 \pm 2.0$ & $0.020 \pm 0.020$ & \\
& 924.599 & $V$ & 5.1 & $0.332 \pm 0.080$ & $-0.379 \pm 0.080$ & $0.504 \pm 0.080$ & $155.6 \pm 4.5$ & $0.027 \pm 0.057$ \\
& 924.605 & $R_{\mathrm{C}}$ & & $0.354 \pm 0.039$ & $-0.426 \pm 0.039$ & $0.554 \pm 0.039$ & $154.9 \pm 2.0$ & $-0.051 \pm 0.028$ & $1.8 \sigma$ \\
& 924.612 & $I_{\mathrm{C}}$ & & $0.373 \pm 0.026$ & $-0.269 \pm 0.026$ & $0.460 \pm 0.026$ & $162.1 \pm 1.6$ & $0.011 \pm 0.018$ & \\
& 929.619 & $V$ & 5.2 & $0.261 \pm 0.091$ & $-0.252 \pm 0.091$ & $0.363 \pm 0.091$ & $158.0 \pm 7.2$ & $-0.047 \pm 0.064$ \\
& 929.626 & $R_{\mathrm{C}}$ & & $0.181 \pm 0.040$ & $-0.356 \pm 0.040$ & $0.399 \pm 0.040$ & $148.5 \pm 2.9$ & $0.037 \pm 0.028$ \\
& 929.633 & $I_{\mathrm{C}}$ & & $0.348 \pm 0.029$ & $-0.427 \pm 0.029$ & $0.427 \pm 0.029$ & $162.3 \pm 1.9$ & $-0.039 \pm 0.020$ & $2.0 \sigma$ \\
929.645 & $V$ & 5.1 & $0.200 \pm 0.088$ & $-0.585 \pm 0.088$ & $0.618 \pm 0.088$ & $144.4 \pm 4.1$ & $0.068 \pm 0.060$ & \\
& 929.652 & $R_{\mathrm{C}}$ & & $0.192 \pm 0.040$ & $-0.431 \pm 0.040$ & $0.472 \pm 0.040$ & $147.0 \pm 2.4$ & $0.005 \pm 0.028$ & \\
& & & & & & & &
\end{tabular}
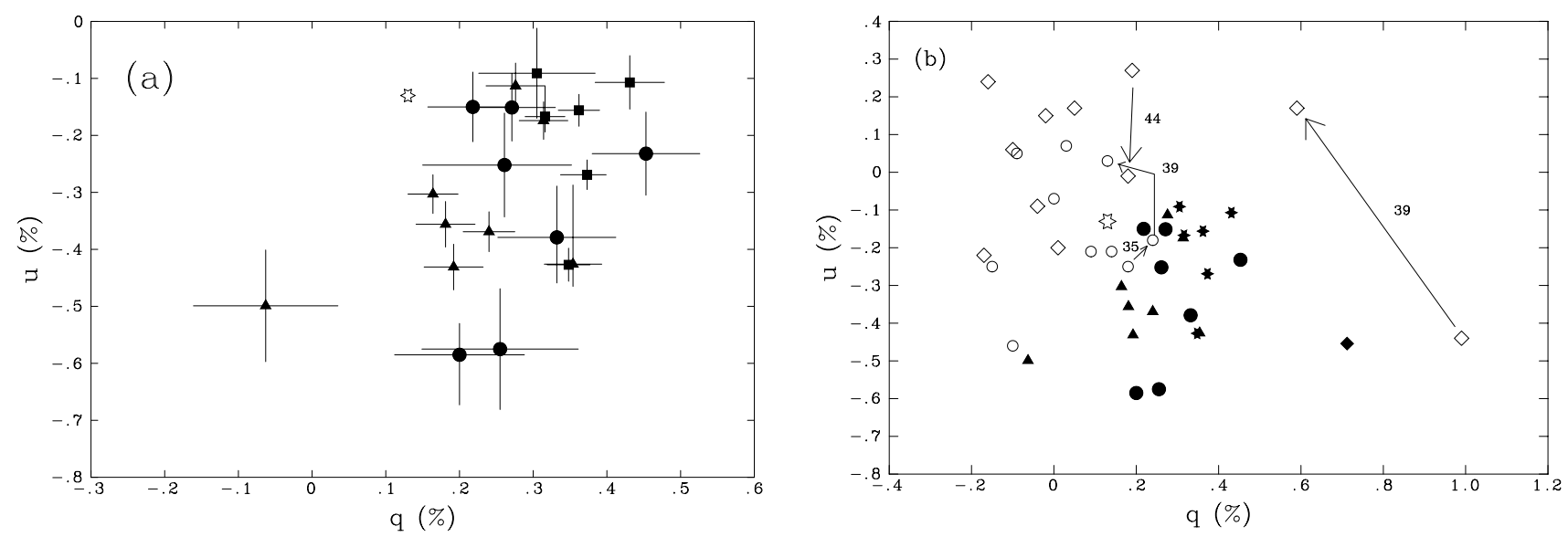

Fig. 1. a) $q u$ diagram for $\mathrm{R} \mathrm{Scl}$; data from this paper. Filled circles: $V$-band; filled triangles, $R_{\mathrm{C}}$-band; filled squares, $I_{\mathrm{C}}$-band measurements. b) $q u$ diagram for $\mathrm{R} \mathrm{Scl}$ based on our data combined with those of Serkowski \& Shawl (2001). Filled symbols, data from this paper, open symbols, data from Serkowski \& Shawl. Diamonds, $B$-band; circles, $V$-band; triangles, $R_{\mathrm{C}}$-band; stars, $I_{\mathrm{C}}$-band. Arrows connect consecutive measurements, with time interval in days as indicated. On both figures the open star is our estimate of IS polarization in the $V$-band. See text for details.

that, in many cases, the polarization vector has a tendency to rotate around the $q u$-point of the IS polarization, while also being variable in magnitude. There is no evidence that $\mathrm{R} \mathrm{Scl}$ is a binary, although its long-tem ( $\gtrsim 3000$ days) light curve is strikingly similar to that of the post-AGB binary U Mon, which has 2600 day orbital and photometric period (van Winckel et al. 1999). However it is highly unlikely that the polarization (and the polarimetric variations) of $\mathrm{R} \mathrm{Scl}$ are related to binarity as the variations occur on timescales that are «one pulsational period. Indeed, at first sight the behaviour we see in Fig. 1b seems consistent with scattering on dust condensations rotating in a CS envelope. The data points fill the $q u$-plane more-or-less uniformly, as we might expect for a nearly face-on disc.

To explore this possibility we consider a simple model of a condensation containing $N$ identical grains moving in a circular orbit of radius $R$ around the star (see Fig. 2; note that a similar model has been discussed by Code \& Whitney 1995 in the context of RCB stars). Unpolarized light from the star, assumed to be a point source, is scattered to the observer and we can in principle calculate the resulting polarization 


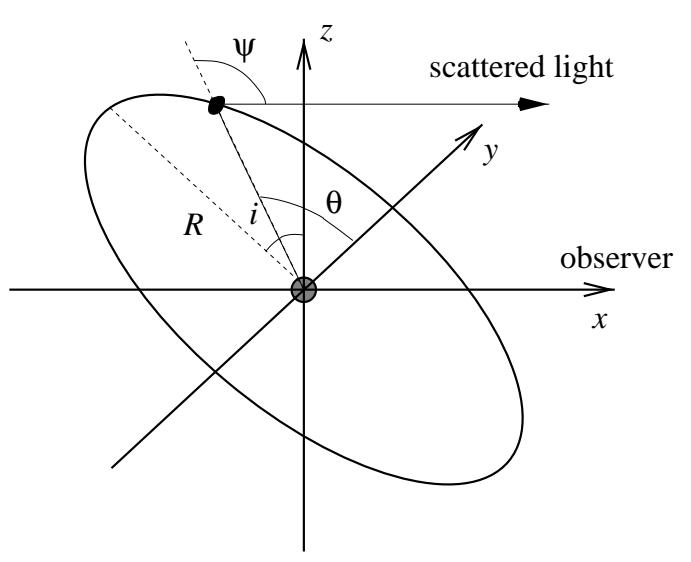

Fig. 2. Simple model for light scattering by a small cloud of dust in a circular orbit of radius $R$ inclined at angle $i$ to the plane of the sky. The observer is located along the $x$-axis and the $y z$-plane defines the plane of the sky; north is along the $z$-axis. See text for details.

as a function of location in the orbit. Assuming for simplicity that there is no internal extinction in the cloud, and that the star-cloud system is unresolved, the polarization in the point source approximation is

$P=\frac{\alpha\left(\iota_{1}-\iota_{2}\right)}{\alpha\left(\iota_{1}+\iota_{2}\right)+1}$,

where $\iota_{1}(\psi)$ and $\iota_{2}(\psi)$ are related to the amplitude functions for scattered radiation (see van de Hulst 1957), $\alpha=N \lambda^{2} / 8 \pi^{2} R^{2}$ and $\lambda(=0.55 \mu \mathrm{m})$ is the wavelength. The scattering angle $\psi=$ $\cos ^{-1}\{\sin \theta \sin i\}$, where $i$ is the inclination of the orbit to the plane of the sky and $\theta$ is the angular position in the orbit (see Fig. 2).

We first note from Eq. (1) that $P$ is independent of the stellar radiation; therefore even if the star (as does $\mathrm{R} \mathrm{Scl}$ ) varies this mechanism will not give rise to polarimetric variations that are correlated with light curve phase. While the intensity of the scattered light - and hence $q, u$ and the degree of polarizion - must change as the star varies, there can be no variation in the observed polarization if the geometry of the system is static (i.e. the relative locations of star and scattering condensation(s) remain unchanged); it is the variation of $\psi$ in Eq. (1) that gives rise to variations in polarization. Likewise, occultation effects, in which inferior condensations obscure the star from the orbiting condensation, will not affect the observed polarization.

To determine the locus in the $q u$ plane for an orbiting condensation we consider amorphous carbon grains for which we take optical constants from Zubko et al. (1996). We shall see below (Sect. 3.2) that the wavelength-dependence of polarization requires small grains, and we assume grain radius $a=$ $0.01 \mu \mathrm{m}$; we have carried out similar calculations for $0.01 \mu \mathrm{m}$ silicon carbide particles, with similar results. The loci in the $q u$ plane, for orbital inclinations $30^{\circ}$ and $70^{\circ}$, are shown in Fig. 3 . For this figure we have taken

$\alpha \equiv \frac{N \lambda^{2}}{8 \pi^{2} R^{2}}=10^{6}$

(see above), so that there are $N \sim 4.6 \times 10^{43}\left(R / 600 R_{\odot}\right)^{2}$ grains, or a grain mass $\simeq 6.4 \times 10^{-3}\left(R / 600 R_{\odot}\right)^{2} a_{0.01}^{3} M_{\oplus}$, in the condensation; the density of the condensation $\rho_{\mathrm{c}}$ is thus $\sim 2.7 \times 10^{-9}\left(r_{\mathrm{c}} / \mathrm{AU}\right)\left(R / 600 R_{\odot}\right)^{2} a_{0.01}^{3} \mathrm{~kg} \mathrm{~m}^{-3}$, where $r_{\mathrm{c}}$ is the condensation radius, $a_{0.01}$ is grain radius in units of $0.01 \mu \mathrm{m}$ and we have assumed a gas-to-dust ratio of 100 .

We see from Fig. 3 that, in the course of a single orbit, the polarization makes two circuits of the $q u$ plane. Also, to a good approximation, the orbit inclination $\simeq \arctan (\Delta u / \Delta q)$, where $\Delta q, \Delta u$ are the ranges of the $q u$ values. In principle, therefore, with sufficient $q u$ data, it might be possible to estimate the inclination of any preferred plane of the CS environment of R Scl; however the data we currently have at our disposal do not allow us to do so.

However we can use Fig. 3 to place limits on the value of $\alpha$ for the condensation. If we use $\alpha \simeq 10^{7}$, the calculated values of $\Delta q$ and $\Delta u$ are $\sim 5 \%$, much larger than observed; on the other hand, $\alpha \simeq 10^{5}$ gives $\Delta q$ and $\Delta u \sim 0.02 \%$. We conclude therefore that $\alpha \simeq 10^{6 \pm 1}$.

Clearly there will be more that one condensation in the CS environment of $\mathrm{R} \mathrm{Scl}$, and so a direct comparison of Fig. 1a (which covers about 1 pulsational cycle) and 3 should be approached with caution. However if we consider the locus of the polarization vector in the $q u$-plane for the $\mathrm{R} \mathrm{Scl}$ data obtained within one pulsation period (see Fig. 1), we estimate the orbital quasi-period to be in the range $\approx 470^{\mathrm{d}}$ to $\approx 1600^{\mathrm{d}}$, clearly longer than the period of pulsation. For some of the dates the polarization vector does not change in respect of PA in the $q u$-plane, but does vary in magnitude (see, for example, our $V$-band data for JD 575-579).

From the quasi-period of possible rotation of the dust condensations of $\sim 470 \mathrm{~d}-1600^{\mathrm{d}}$ and assuming Keplerian motion, we estimate that the condensations are located at a distance of about $R \sim 1.1-2.7\left(M / M_{\odot}\right)^{2 / 3} \mathrm{AU} \simeq 1.8-4 \mathrm{AU}$ if the orbits are circular. The bolometric luminosity of $\mathrm{R} \mathrm{Scl}$ indicates a stellar radius of $R_{\star} \sim 600-700 R_{\odot}$, which is of the same order as the deduced $R$ unless the mass of $\mathrm{R} \mathrm{Scl}$ is much larger $\left(\gtrsim 10 M_{\odot}\right)$ than we have estimated. We consider this unlikely, so we therefore conclude that the orbits of the condensations are highly eccentric.

Lorenz-Martins et al. (2001), on the basis of IRAS data, have concluded that the inner radius of the dust shell around $\mathrm{R} \mathrm{Scl}$ is $\sim 5 R_{\star}$. Furthermore, according to Le Bertre (1988a,b), dust formation takes place at a distance $\sim 6 R_{\star}$ from the stellar surface, and Danchini et al. (1994) estimate the inner dust shell radii for the dust shells of several carbon-rich stars to be $\sim 2-7 R_{\star}$. Thus if the condensations are in highly eccentric orbits, our interpretation of the polarimetric variations of $\mathrm{R} \mathrm{Scl}$ is therefore in line with these estimates of the parameters of the CS dust shell of R Scl, obtained independently from infrared data.

We note that the mass of the CS shell of $\mathrm{R} \mathrm{Scl}$ is $\sim 0.01 M_{\odot}$ (e.g. Gonzáles Delgado et al. 2001). Our deduced condensation mass of $\sim 0.64 M_{\oplus}$ might therefore imply $\sim 500$ condensations. However such a large number would cleary "smear out" any polarimetric variations, and in any case does not allow for the presence of a relatively "smooth" background of dust. We therefore conclude that the number of condensations is $\ll 500$. 

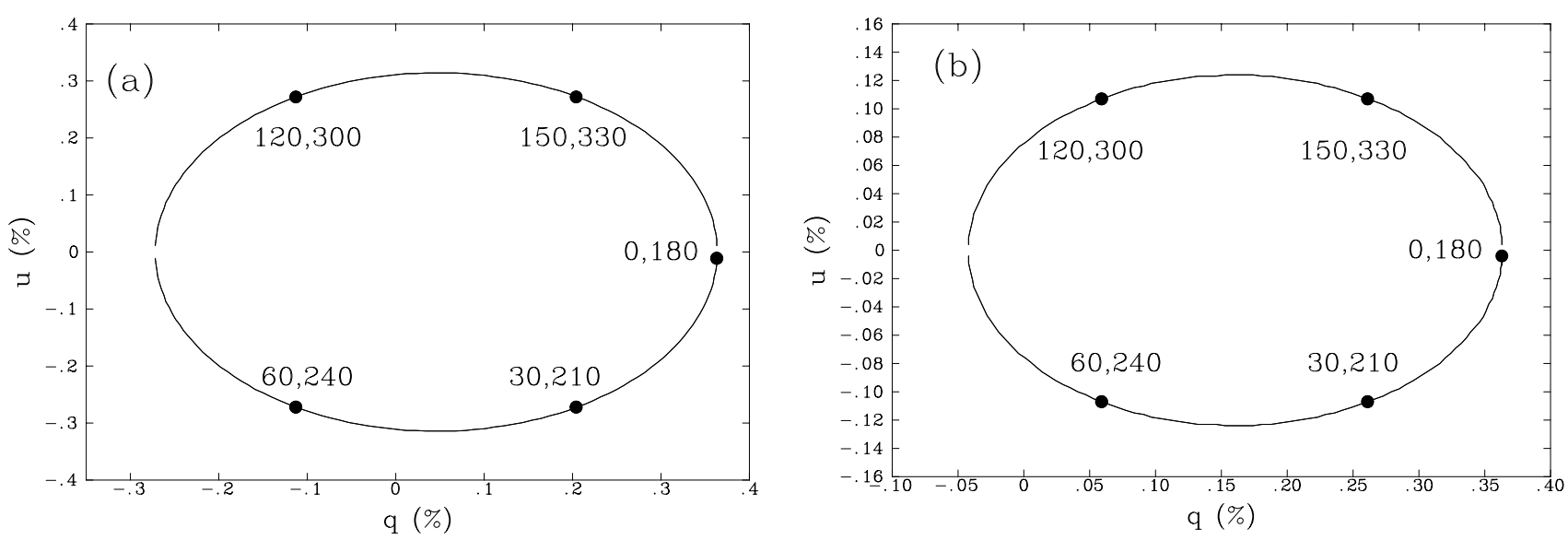

Fig. 3. a) Variations in $q u$ plane for condensation consisting of $0.01 \mu \mathrm{m}$ amorphous carbon grains in circular orbit inclined at $30^{\circ}$ to plane of sky; points are at intervals of 30 days, as indicated. b) As a) but for inclination $70^{\circ}$. See text for details.

\subsubsection{Rapid variability}

We now consider the rapid variability of the Stokes parameters that we see on a short time-scale (hours-days). As already noted, these can not be due to variable illumination by the star; they must reflect either variable illumination of dust condensations as they rise or fall in the CS environment, or form and disperse. The time-scale for rising or falling in the CS environment is likely to be too long to account for the observed rapidity of the polarimetric variations, and formation and/or dispersal is more likely.

One possible dispersal mechanism is tidal disruption. A dust condensation of density $\rho_{\mathrm{c}}$ will be tidally dispersed on a time-scale (Donnison \& Williams 1975)

$\tau \simeq \frac{\mathcal{P}}{3 \pi} \frac{x^{3 / 2}}{1-x^{3}}$,

where $\mathcal{P}$ is the orbital period, $x=R / R_{\mathrm{L}}$ and $R_{\mathrm{L}}$ is the Roche limit

$R_{\mathrm{L}} \simeq 1.52\left(\frac{M}{\rho_{\mathrm{c}}}\right)^{1 / 3}$

for stellar mass $M$. For a $3.5 M_{\odot}$ star and the condensation density deduced in the previous section, we find $R_{\mathrm{L}} \simeq 2.1 \times 10^{13} \mathrm{~m}$ or $\sim 50 R_{\star}$, a result that scales linearly with the dimensions of the condensation. Thus $x \simeq 0.15\left(r_{\mathrm{c}} / \mathrm{AU}\right)$ and

$\frac{\tau}{\text { days }} \simeq 6\left(\frac{P}{1000 \text { days }}\right)\left(\frac{x}{0.15}\right)^{3 / 2}$.

As the condensation disperses, Eqs. (1) and (2) lead us to expect changes in polarization on a time-scale $\sim \tau$; the observed time-scale of $\lesssim 1$ day implies condensations of dimensions $\sim 2.3 \mathrm{AU}$. Combining this with the condensation properties from the previous section and extinction efficiency $\simeq 10^{-6}$ for $0.01 \mu \mathrm{m}$ amorphous carbon grains, we find that the extinction through a condensation is typically $\sim 1$ mag in the $V$-band.

The frequent formation of dust in the CS environments of carbon stars is a common property of these objects (see Busso et al. 1999; Winters et al. 1995). Our observations reinforce the important role played by dust scattering in the CS envelopes to account for their polarimetric characteristics. In this context we note the work of Gonzáles Delgado et al. (2001), who report the first optical images of scattered light from gas/dust shells around $\mathrm{R} \mathrm{Scl}$. These authors noted that these shells have an overall spherical symmetry, but with non-uniform surface brightness indicating a highly clumped medium. Clearly polarization can not occur in a uniform spherically symmetric envelope; our interprepation of polarimetric variability in terms of the motion of dust condensations in highly eccentric orbits is therefore consistent with the observations of Gonzáles Delgado et al.

It was also suggested by Gonzáles Delgado et al. (2001) that some features in the $\mathrm{KI}$ and $\mathrm{NaD}$ lines are due to Rayleigh scattering by small grains, again in good agreement with our conclusions. The time-scale for polarimetric variations is consistent with the scattering dust located close to the star $(R \sim$ $\left.5 R_{\star}\right)$, i.e. in the inner zone of a warm shell. This would be consistent with either the injection of a dust condensation into the envelope, or equivalently the dispersal of dust condensations as considered above. The typical time-scale for the latter behaviour certainly seems consistent with the observations.

The other behaviour seen in Fig. $1 b$ is that most of our data are located in the first and fourth quadrants of the $q u$ plane, centered on the IS polarization point, whereas most of the data from Serkowski \& Shawl (2001) are concentrated in second and third quadrants. The observations of R Scl from Serkowski $\&$ Shawl (2001) covered a period of about 3 years (or a few pulsation periods). This behaviour suggests global changes in the CS environment of R Scl, on time-scales of a few pulsation periods.

Finally, we note the marginal detection of circular polarization in $\mathrm{R} \mathrm{Scl}$ in the course of some of our observations. While the detection level for $P_{\text {circ }}$ is not large $(\approx 2 \sigma)$, it is important to note that the mean value of $P_{\text {circ }}$ changed sign for the dates JD571-579 a time, as we noted above, there were changes in the degree of polarization but not in PA. If the change of sign is real, the significance of the result is enhanced, and the circular polarization may be attributed to multiple scattering in newly formed dense dust condensations. 


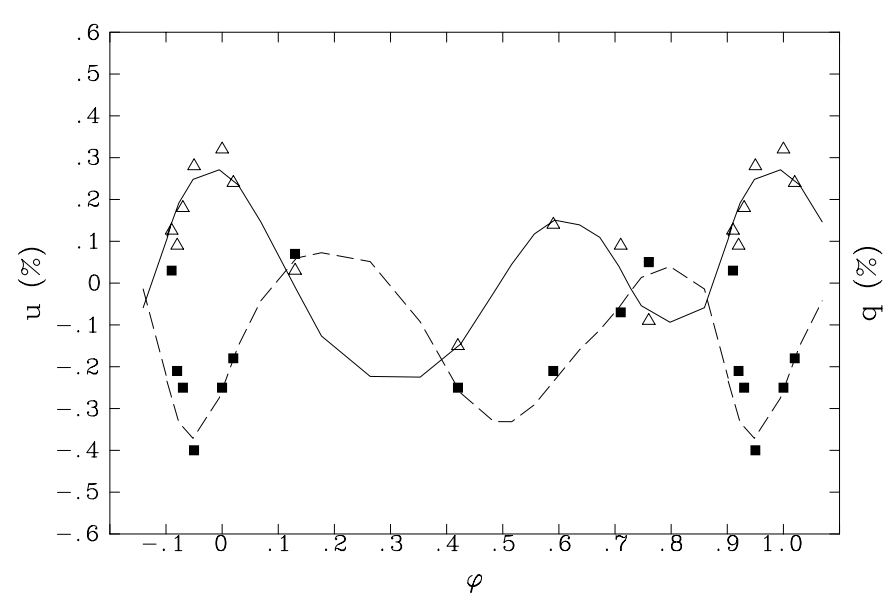

Fig. 4. Relation between $V$-band Stokes parameters and light curve phase variations for $\mathrm{R} \mathrm{Scl}$. Data from this paper and Serkowski \& Shawl (2001). Lines are included to guide the eye. Squares and dashed line, $q$; triangles and solid line, $u$.

\subsubsection{Dependence of polarization on light-curve phase}

In the previous subsection, we attributed the polarimetric variations of $\mathrm{R} \mathrm{Scl}$ within a pulsation period to scattering by small amorphous carbon grains located in condensations in the CS envelope. We noted that, with this interpretation, no dependence on light curve phase is to be expected.

In this Section, however, we use our data, together with those of Serkowski \& Shawl (2001), to investigate the possible long-term dependence of polarization on pulsation period. We estimate phases for the observations reported by Serkowski \& Shawl (2001) using the synthetic light curve calculated by Whitelock et al. (1997); the $m_{V}$ magnitudes in Serkowski \& Shawl (2001) are in excellent agreement with those calculated from the ephemerides. We plot the Stokes parameters for theirs and our $V$-band data against phase in Fig. 4. Although the data set is not large, periodic variations of both Stokes parameters are evident, with a quasi-period $\simeq 170^{\mathrm{d}}-190^{\mathrm{d}}$, about half the pulsation period. We suggest that the intrinsic polarization of $\mathrm{R} \mathrm{Scl}$ becomes higher at phases 0 and 0.5 of the pulsation period, i.e. at maximum and at minimum light. Unfortunately the data at our disposal are too sparse and too poorly sampled to enable us to carry out a Fourier analysis, and this conclusion should therefore be regarded with caution.

However we note from Fig. 4 that the dependence of the Stokes parameters on phase are anticorrelated; the same behaviour (but with smaller amplitudes) was observed for the RS CVn stars GK Hya and UX Ari by Scaltriti et al. (1993). To explain similar behaviour for the carbon-rich post-AGB star AC Her, Henson et al. (1985) suggested nonradial puslations in the stellar atmosphere. Our result may therefore have some bearing on whether R Scl pulsates in "fundamental" or "first overtone" modes (see Sect. 1).

At this point we emphasize that the analysis of polarimetric variability as a function of photometric phase should be carried out only using the Stokes parameters. Figure 4 demonstrates that a comparison of the degree of polarization with phase (as is commonly carried out) is not informative and is likely to lead to erroneous conclusions. Taking into account possible anticorrelation between the Stokes parameters, the degree of polarization may appear essentially constant, while the polarimetric parameters display significant variation.

\subsection{Wavelength-dependence of polarization}

The wavelength-dependence of intrinsic polarization in 1994 and 1995 is depicted in Fig. 5a, where some data obtained through $B V$ filters by Serkowski \& Shawl (2001) are also included. Although the error in our $B$-band measurement is large, a tendency for an increase in the degree of polarization from the $I_{\mathrm{C}}$-band to the $B$-band is evident, suggesting a wavelengthdependence $P \underset{\sim}{\propto} \lambda^{-3}-\lambda^{-4}$; one (limited) dataset in Serkowski \& Shawl (2001; see Fig. 5a) is also consistent with this wavelength-dependence.

This wavelength-dependence strongly hints at scattering by small particles as the polarization mechanism. Indeed, the fact that the values of $P_{\mathrm{B}}$ in the data of Serkowski \& Shawl (2001) for most of their observations are significantly and consistently higher than the corresponding $P_{\mathrm{V}}$ values is consistent with the dust scattering interpretation. To explore this hypothesis we again use the fact that $\mathrm{R} \mathrm{Scl}$ is a carbon star and consider scattering by $0.01 \mu \mathrm{m}$ amorphous carbon grains, with optical constants from Zubko et al. (1996). The results for inclination $30^{\circ}$ and $\theta=60^{\circ}$ are shown in Fig. 5b. We see that the general wavelength-dependence seen in Fig. 5a is indeed consistent with scattering by small amorphous carbon particles.

We also note from Table 1 that there is no evidence for a dependence of position angle on wavelength. This strongly supports the hypothesis that there is only one polarigenic mechanism at work. However there is marginal evidence that the position angle may vary with epoch, as might be expected as the distribution of condensations in the CS environment changes. This is again consistent with our suggestion that the polarigenic mechanism is scattering by small carbon particles in the CS environment.

\section{Concluding remarks}

(i) We have presented new measurements of the linear and circular polarization of the carbon star $\mathrm{R} \mathrm{Scl}$, and we have analysed its polarimetric variability on different timescales, from hours to a year. There is strong evidence, from polarimetric variability and from the wavelengthdependence of polarization, for the presence of intrinsic polarization.

(ii) The wavelength-dependence of polarization is close to $P \propto \lambda^{-4}$, consistent with Rayleigh scattering by small $(\sim 0.01 \mu \mathrm{m})$ amorphous carbon grains in the CS environment.

(iii) We conclude that the CS medium is highly clumped, and that the polarimetric variations are consistent with scattering by small grains located in condensations of mean density $\sim$ a few $\times 10^{-9} \mathrm{~kg} \mathrm{~m}^{-3}$.

(iv) We have estimated possible quasi-periods of rotation of dust condensations in the CS environment, and have 

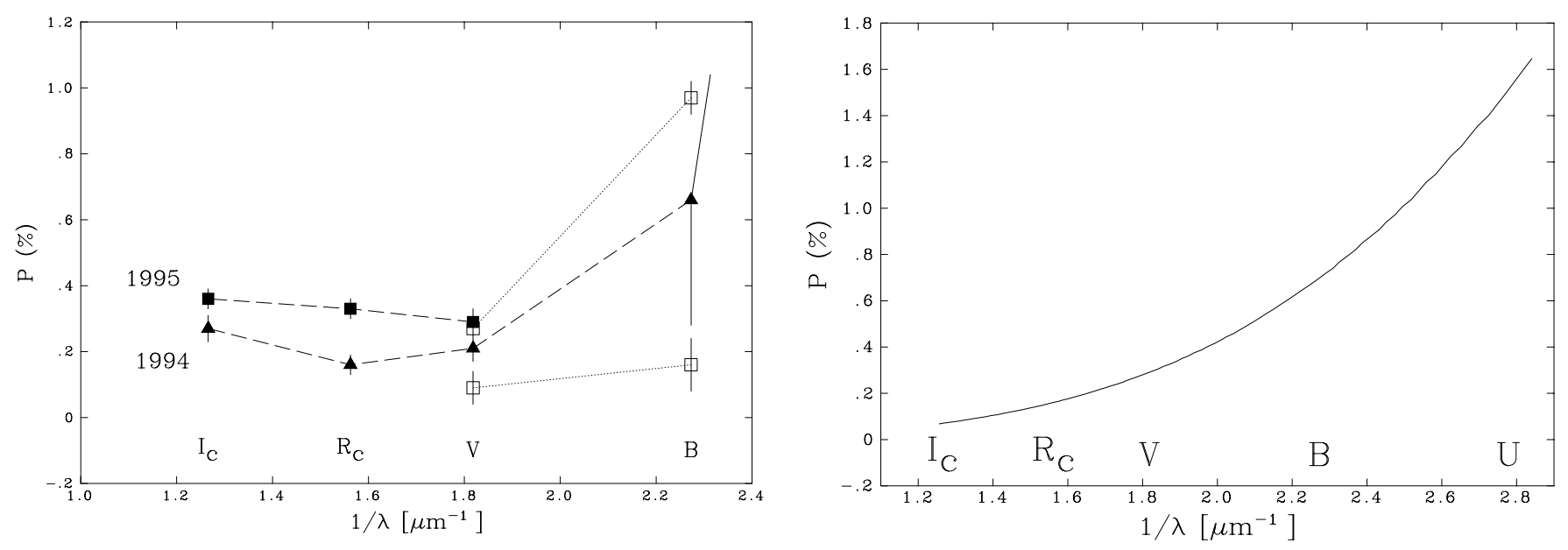

Fig. 5. a) Average wavelength dependence of intrinsic polarization for R Scl in 1994 and 1995 (full points and broken lines); data corrected for instrumental and IS polarization. Open symbols and dotted lines, data from Serkowski \& Shawl (2001) averaged for two different periods; these data also corrected for IS polarization. b) Calculated wavelength-depenence of polarization for $0.01 \mu \mathrm{m}$ amorphous carbon grains; see text for details.

estimated their properties and their location in the CS shell from the polarimetric variations. These estimates are consistent with previous - independent - estimates if the condensations move on highly eccentric orbits.

(v) There is some evidence that the polarimetric variations may be linked with the photometric variations.

Further polarimetric observations are required, covering an entire photometric cycle. These would help to confirm or otherwise the link between polarization and pulsation periods and in particular, resolve the question of the pulsational modes of R Scl. Furthermore, comparison of the data presented here with those given by Serkowski \& Shawl (2001) indicates that major changes in the CS environment of R Scl have occurred over a $\sim 30$ year period. We encourage observers with access to instrumentation having polarimetric capability to monitor the polarization of R Scl. The wavelength-dependence of polarization, and its variation, should also be investigated over a long timebase; this is important to investigate the distribution of CS matter, particularly condensations and inhomogeneities.

Acknowledgements. We thank the referee, Dr Susan Tramell, for helpful and constructive comments on an earlier version of this paper, which uses observations made at the South African Astronomical Observatory (SAAO). We again acknowledge generous awards of time at the SAAO, and the U.K. Particle Physics and Astronomy Research Council (PPARC) for travel funds. We also acknowledge the support of Dr. D. Buckley at SAAO. RVY was supported by a Royal Society Grant, and by grants RFBR 02-02-16543 and INTEGRACIIA. This work made use of the SIMBAD database.

\section{References}

Alksnis, A., Balklavs, A., Dzervitis, U., \& Eglitis, I. 1998, A\&A, 338, 209

Busso, M., Gallino, R., \& Wasserburg, G. J. 1999, APA\&A, 37, 239

Clarke, D., Smith, R. A., \& Yudin, R. V. 1998, A\&A, 336, 604

Clarke, D., \& Stewart, B. G. 1986, Vistas in Astron., 29, 27

Code, A. D., \& Whitney, B. A. 1995, ApJ, 441, 400
Cropper, M. 1985, MNRAS, 212, 709

Danchini, W. C., Bester, M., Degiacomi, C. G., Greenhill, L. J., \& Townes, C. H. 1994, AJ, 107, 1469

Donnison, J. R., \& Williams, I. P. 1975, MNRAS, 172, 257

Gledhill, T. M., Chrysostomou, A., Hough, J. H., \& Yates, J. A. 2001, MNRAS, 332, 331

González Delgado, D., Olofsson, H., Schwarz, H. E., Eriksson, K., \& Gustafsson, B. 2001, A\&A, 372, 885

Heiles, C. 2000, AJ, 119, 923 (vizier.u-strasbg.fr/vis-bin/Cat?II/226)

Henson, G. D., Kemp, J. C., \& Kraus, D. J. 1985, PASP, 97, 1192

Hron, J., Loidl, R., Hoefner, S., et al. 1998, A\&A, 335, L69

Johnson, J. J., \& Jones, T. J. 1991, A\&A, 101, 1735

Le Bertre, T. 1988a, A\&A, 190, 79

Le Bertre, T. 1988b, A\&A, 203, 85

Lorenz-Martins, S., de Arazjo, F. X., Codina Landaberry, S. J., de Almeida, W. G., \& de Nader, R. V. 2001, A\&A, 367, 189

Magalhães, A. M., Coyne, G. V., Codina-Landaberry, S. J., \& Gneiding, C. 1986, A\&A, 154, 1

Monnier, J. D., Tuthill, P. G., \& Danchi, W. C. 2000, ApJ, 545, 957

Olofsson, H., Bergman, P., Eriksson, K., \& Gustafsson, B. 1996, A\&A, 311, 587

Olofsson, H., Bergman, P., Lucas, R., et al. 2000, A\&A, 353, 583

Raveendran, A. V., \& Rao, N. K. 1989, A\&A, 215, 63

Scaltriti, F., Piirola, V., Coyne, G. V., et al. 1993, A\&AS, 102, 343

Serkowski, K., \& Shawl, S. J. 2001, AJ, 122, 2017

van de Hulst, H. C. 1957, Light Scattering by Small Particles (Dover) van Winckel, H., Waelkens, C., Fernie, J. D., \& Waters, L. B. F. M. 1999, A\&A, 343, 202

Vassiliadis, E., \& Wood, P. R. 1993, ApJ, 413, 641

Whitelock, P. A., Feast, M. W., Marang, F., \& Overbeek, M. D. 1997, MNRAS, 288, 512

Winters, J. M., Fleischer, A. J., Gauger, A., \& Sedlmayr, E. 1995, A\&A, 302, 48

Yudin, R. V., \& Evans, A. 1998, A\&AS, 131, 401

Yudin, R. V., \& Evans, A. 2002, A\&A, 386, 916

Zubko, V. G., Mennella, V., Colangeli, L., \& Bussoletti, E. 1998, MNRAS, 282, 1321 